\title{
Coherent Tunneling through Quantum Wire Tailored by Gaussian Profile
}

\author{
Hassan S. Ashour \\ Department of Physics, Al-Azhar University-Gaza, Palestine \\ E-mail: hashour@alazhar-gaza.edu \\ Received December 31, 2010; revised January 5, 2011; accepted February 2, 2011
}

\begin{abstract}
In this paper, we propose a novel structure of quantum waveguide. In this structure we tailored the quantum wire by Gaussian Profile. Thus, the Dirac-Delta function potentials are weighted according to Gaussian distribution function. We studied the electronic transmission properties through this tailored quantum waveguide structure. We have assumed that single free-electron channel is incident on the structure and the scattering of electrons is solely from the geometric nature of the problem. We have used the transfer matrix method to study the electron transmission. Coherent Tunneling is achieved through this structure, which is well-defined allowed conduction bands. The electronic conductance spectrum depends on the number of the Dirac delta function potential in the quantum wire. When the number of Dirac delta function potentials in the structure and their strengths are increased, both well defined conductance bands and sharper and narrower forbidden bands are formed. This novel structure has a good defect tolerance. The structure tolerates strength defect and tolerates position defect for the central Dirac delta function in the Gaussian distribution.
\end{abstract}

Keywords: Mesoscopic Systems, Quantum Waveguide, Quantum Wire

\section{Introduction}

In recent years, the electronic conductance through nano-structure grabs many researchers attention for their potential applications [1-9]. Moreover, there was an interest in coherent electronic tunneling through onedimensional scattering problems, especially in those cases where the potential is periodic structure with finite number of identical cells $[10,11]$. Because of the remarkable advances in nano-technology and micro fabrication, it is possible to confine electrons in a conductor with a lateral extent of $100 \mathrm{~nm}$ or less, resulting narrow quantum wire [12]. In these mesoscopic devices, the electron transport is best described by quantum mechanics. Miniature size of these devices eliminates the defect of scattering. At a low enough temperature, the motion of electrons through these devices is ballistic or quasiballistic and the electron-phonon interaction can be neglected. Therefore, the phase coherence length enlarges enough when compared with the device dimension. Mesoscopic devices can be considered as coherent elastic scatterer [12]. Therefore, the electron transport properties solely depend upon the geometrical structure of the quantum waveguide.
In recent years, many methods have been used to achieve coherent tunneling through their electronic devices, i.e. tunneling magnetoresistance (TMR) device $[13,14]$. Recently, Ashour et al. [15] had achieved coherent tunneling through their proposed a novel structure, which is the binomially tailored waveguide quantum wire in which each Dirac-Delta function potential-strength is weighted according to the binomial distribution law. In this paper, we are going to carry this reshaping of the quantum wire further, by introducing quantum wire tailored by Gaussian profile.

This paper is organized as follow: in Section 2, we outline the transfer matrix method which connects the solutions at the ends of the waveguide quantum wires. In Section 3, we introduce the novel structure of the QW tailored by Gaussian Profile. In Section 4, we explore the effect of the potential strength of the central Dirac Delta potential, and the number of Dirac Delta potentials in the quantum wire on the electrical conductance. In Section 5, we explore defect effects on the electronic conductance spectrum through the Gaussian profile tailored quantum wire; we are going to address two defects namely: dislocation defect of the central Delta potential, and strength 
defect. Section 6 is solely devoted to the conclusions of this study.

\section{Transmission through Periodic Structure}

In this context, we consider a finite periodic structure of Dirac delta function potential, Dirac Comb. We assumed that the structure is narrow enough so that, only a single channel of electrons can be considered. Besides, we neglected electron-electron interaction, and we assumed that the temperature is low enough so that, electron-phonon interaction can be neglected as well. We assumed that the scattering of electrons mainly from the geometrical structure of the potential. The potential can be written as follows:

$$
V(x)=\sum_{j=1}^{N} U_{j} \delta\left(x-x_{j}\right)
$$

Where, $U_{j}$ and $x_{j}$ represent the strength and the position of the delta function respectively, and $N$ is the number of the Dirac delta functions in Dirac Comb. The distance between the adjacent barriers is given by $d_{j}=x_{j+1}-x_{j}$. The Schrödinger wave equation of one dimension can be written as follows:

$$
-\frac{\hbar^{2}}{2 m^{*}} \frac{\mathrm{d}^{2} \psi(x)}{\mathrm{d} x^{2}}+V(x) \psi(x)=E \psi(x)
$$

Where, $V(x)$ is the periodic potential given by Equation (1), $m^{*}$ is the electron effective mass, which is considered approximately constant over the interaction range. The solution of Schrödinger wave equation for single Delta function potential can be found in the literature and also in the transfer matrix formulism [16-19]. The transfer matrix for periodic structure has been used also to study the transmission of electron through Comb struc- ture [16-22]. The transfer matrix, which is related to the input electron wave and the output, is given by [20-22]

$$
\left(\begin{array}{l}
\boldsymbol{C} \\
\boldsymbol{D}
\end{array}\right)=\left(\begin{array}{cc}
\mathrm{e}^{i k x_{j}} & 0 \\
0 & \mathrm{e}^{-i k k_{j}}
\end{array}\right)^{-1}\left(\begin{array}{cc}
1-i \beta_{j} & -i \beta_{j} \\
+i \beta_{j} & 1+i \beta_{j}
\end{array}\right)\left(\begin{array}{cc}
\mathrm{e}^{i k x_{j}} & 0 \\
0 & \mathrm{e}^{-i k x_{j}}
\end{array}\right)\left(\begin{array}{l}
\boldsymbol{A} \\
\boldsymbol{B}
\end{array}\right)
$$

Thus, $\beta_{j}$ is $\gamma_{j} / 2 k$, where $\gamma_{j}$ is $2 m^{*} U_{j} / \hbar^{2}$, and $k$ is the wave number given by $\sqrt{2 m^{*} E / \hbar^{2}}$. So that the transfer matrix at a given single barrier can be written as,

$$
M_{j}=S^{-1}\left(i k x_{j}\right) \cdot \Gamma\left(\beta_{j}\right) \cdot S\left(i k x_{j}\right)
$$

The total transfer matrix which represents the electron propagating through the entire device is just the repetitive product of the transfer matrix of a single barrier. We find

$$
M_{t}=S^{-1}\left(i k d_{N}\right) \cdot \Gamma\left(\beta_{N}\right) \cdot\left[\prod_{j=N-1}^{1} S\left(i k d_{j}\right) \cdot \Gamma\left(\beta_{j}\right)\right] \cdot S(0)
$$

Thus, $d_{j}$ is the periodic spacing between two adjacent Dirac Delta functions.

Then the transmission amplitude is given by [23],

$$
T=\frac{1}{M_{t}(2,2)}
$$

Thus, $M_{t}(2,2)$ is the second element in the second row in a $2 \times 2$ matrix. According to the LandauerButtiker formula, the electron conductance through this structure is $[24,25]$

$$
G=\frac{2 \mathrm{e}^{2}}{h}|T|^{2}
$$

We assume a dimensionless strength for Delta function potential [26] by rescaling our parameters, $\Omega_{j}=m d_{j} U_{j} / \pi^{2} \hbar^{2}$. In Figure 1, we show the con-

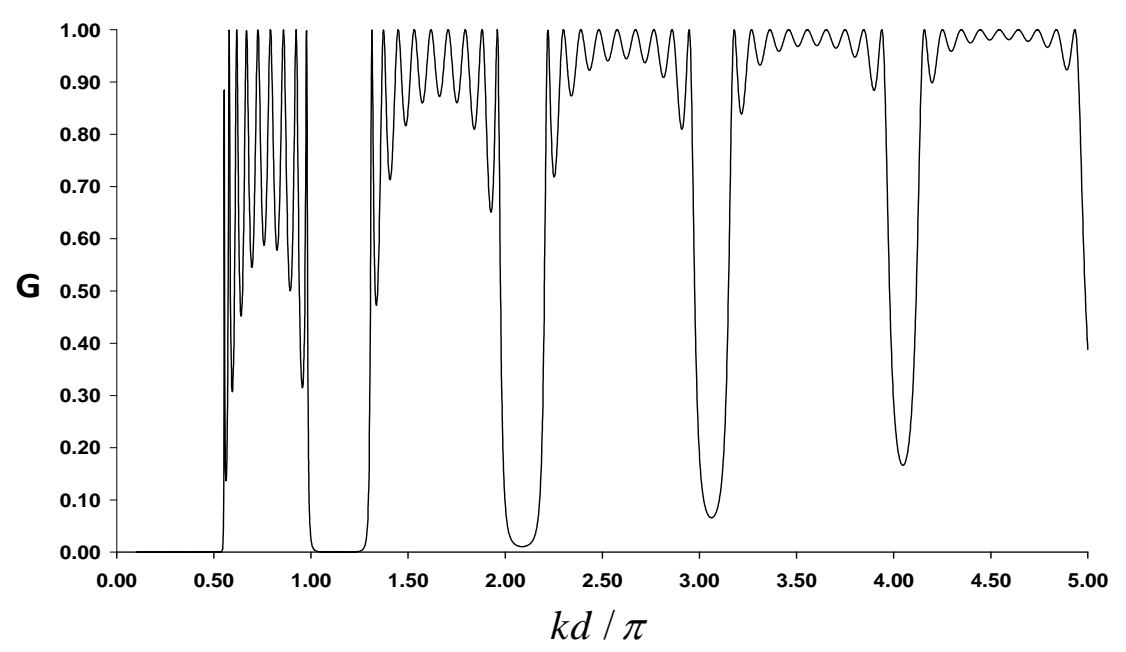

Figure 1. Conductance spectrum $\mathbf{G}$ in the units of $2 e^{2} / h$ as a function of $k d / \pi$ for a sequence of Dirac delta function potential with $N=10$. The strength of the potential here is $\Omega=0.2$. 
ductance through $N=10$ Dirac delta function potential with strength $\Omega=0.2$. A perfect transmission in this case is in general impossible as predicted by $[22,27]$. According to reference [26] we cannot have a resonant transmission, $T=1$, even if $N$ is very large.

\section{Tailored Quantum Wire by Gaussian Profile}

In this subsection, we introduce the new quantum wire structure which is shown in Figure 2. The Dirac delta function has been equally spaced but their strength, $\Omega_{j}$, has been weighted according to the binomial distribution law, which is

$$
\begin{aligned}
& \Omega\left(N_{j}\right)=\frac{1}{\sqrt{2 \pi N p q}} \exp \left(\frac{-\left(x-N_{j} p\right)^{2}}{2 N_{j} p q}\right), \\
& N_{j}=0,1, \cdots, N \text {, and } p+q=1
\end{aligned}
$$

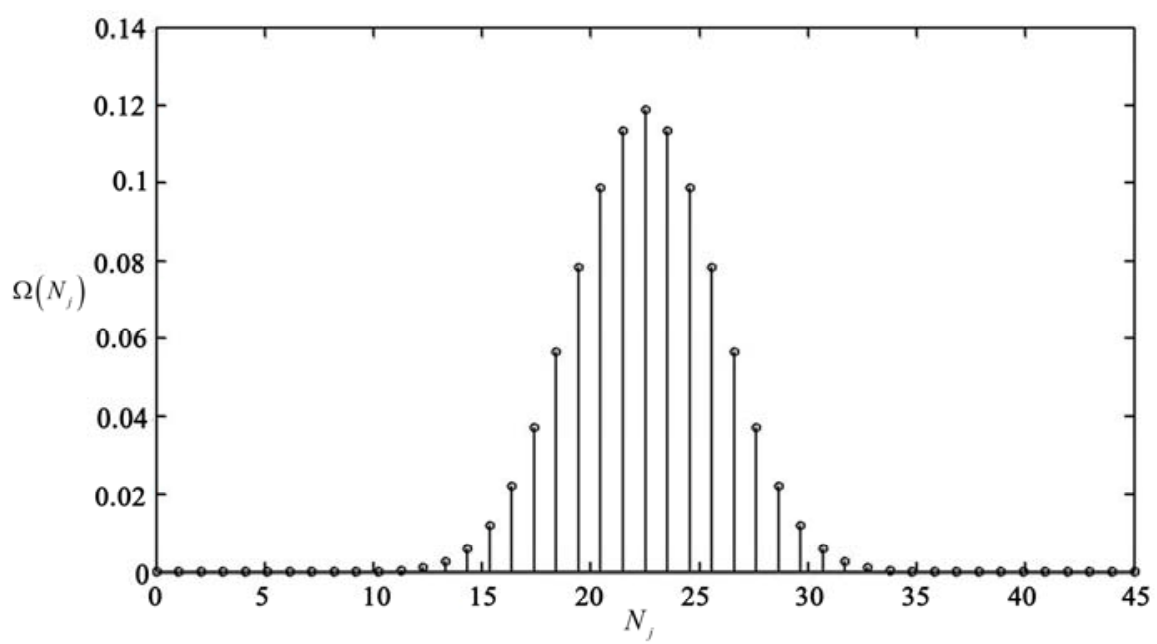

Figure 2. Shows Gaussian tailored Dirac comb potential. $N_{j}$ 's values are weighted according to Equation (8).

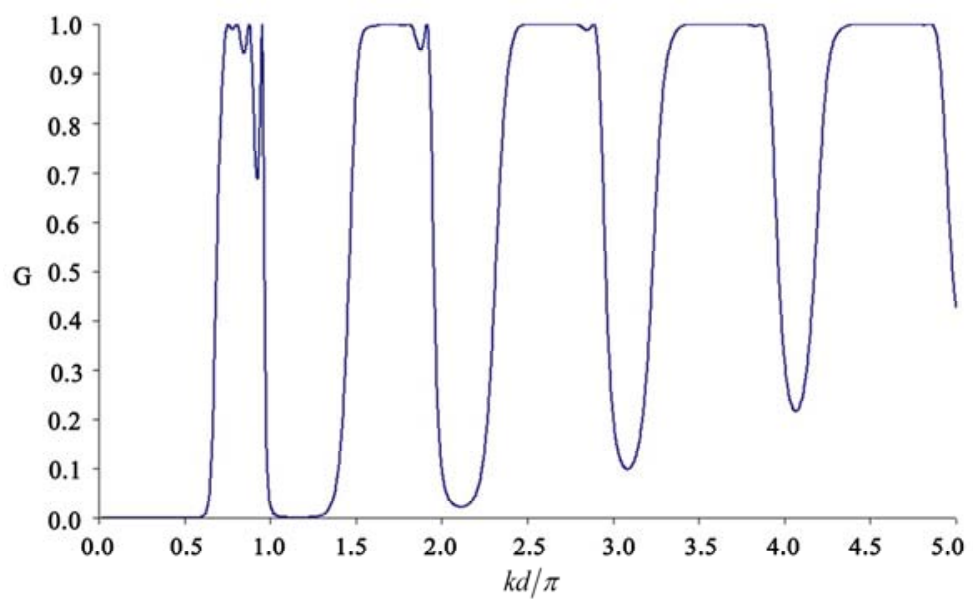

Figure 3. Conductance spectrum $G$ in the units of $2 e^{2} / h$ as a function of $k d / \pi$ for a quantum wire tailored by Gaussian distribution function, with $N=9$. 
tance spectrum through a sequence of a binomially tailored Dirac delta function potentials. It is quite interesting to notice that we have reached a transmission through this structure approaches to unity in the allowed band region without any ripples after some $k$ value. Here, we have a resonant tunneling due to coherent interference effects due to elastic scattering of electrons, which leads the transmission to reach unity and also to have constant value over the allowed band or conduction band. Also, we see that there is a forbidden band or conduction gap where the transmission is small.

\section{4. $N_{j}$ and $\Omega\left(N_{j}\right)$ Effect on Electronic Conductance}

\subsection{Effect of the Number of Dirac Delta Function, $N_{j}$, in the Distribution}

Here, we study the effect of the number of Dirac delta function potential in the Gaussian distribution profile on the electronic conductance through the quantum wire. In Figure 4, we plot the electronic conductance for different number of the Number of Dirac delta function potentials in the Gaussian distribution profile. The electronic conductance spectrum shows flat allowed bands, which were sometimes called, the conductance band, due to coherent tunneling or constructive interference of electronic plane waves and forbidden bands due to total destructive interference of the electronic plane waves. When the Number of Dirac delta function potential, in the Gaussian distribution profile quantum wire, is increased the width of the conduction band increases. The increase in width of the conduction can be explained as follow: the number of electron waves that interfere constructively increases which leads to wider conduction band. This behavior is illustrated in Figure 4, when we compare the electronic conductance taken from the Gaussian profile tailored quantum wires (GTQW), with $N_{j}=17$ and 9 Dirac delta functions potentials. The quantum wire with $N_{j}=17$ Dirac delta function potentials has a wider conduction bands and narrower forbidden bands than the quantum wire with $N_{j}=9$ Dirac delta function potentials.

\subsection{Effect of the Strength of Dirac Delta Function in the Distribution}

In this subsection, we study the effect of the strength of the Dirac delta function potentials on the electronic conductance. In Figure 5, we plot the electronic conductance spectrum for $N_{j}=17$ evaluated according to Equation (9), the curve with squares, and we plot the electronic conductance spectrum for the same number of Dirac delta function potentials but with scaling factor of three. That is, we multiply each Dirac delta function potential by three. The conduction bands turn to be narrower and the forbidden bands wider and well defined. So that, for a well defined electronic conductance, it is better to increase the number of the Dirac delta function potentials in the quantum wire and also to scale up their strength to reach the desired conductance.

\section{Defect Effect}

\subsection{Strength Defect}

In this subsection, we study effect of strength defect of the central element of the GTQW, keeping the other elements and the spacing between the Dirac delta function potentials unaltered, on the electronic conductance

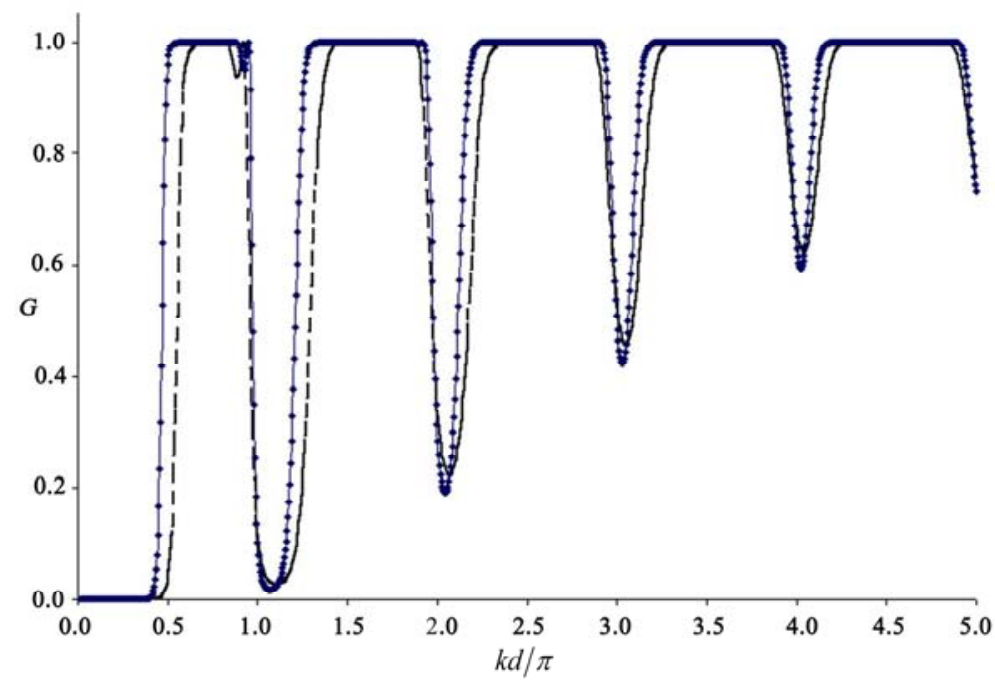

Figure 4. The electronic conductance from two structures: $N_{j}=9$ (dashed line) and $N_{j}=17$. 


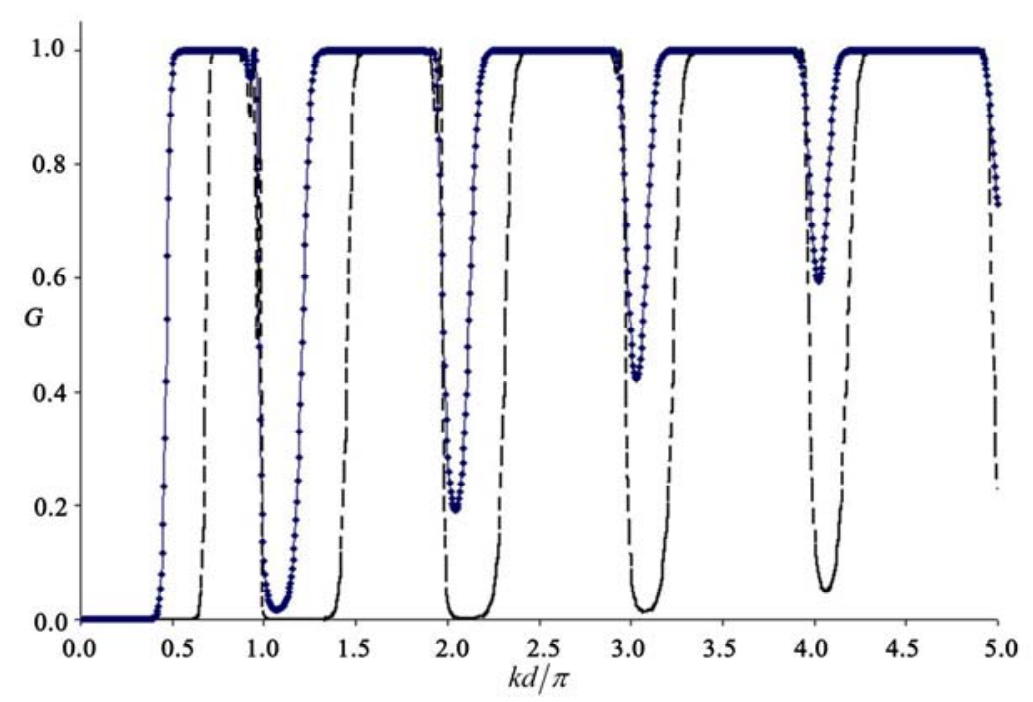

Figure 5. The electronic conductance from a structure of $N_{j}=17$ with different scaling factors, the continuous curve with scaling factor of one and the dashed cure with scaling factor of three.

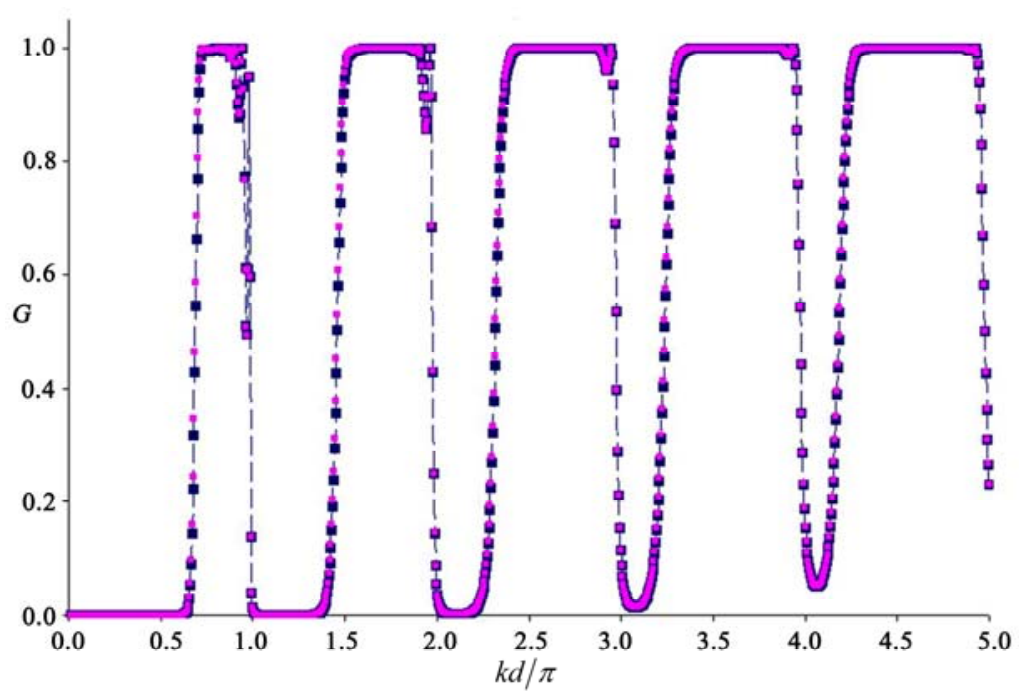

Figure 6. The electronic conductance, in the units of $2 e^{2} / h$ as a function of $k d / \pi$. In this case, the defect is only $\pm 5 \%$, in the strength of the central Dirac delta function.

through the GTQW. We consider the strength defect does not exceed $\pm 5 \%$ of the Dirac delta function potential strength. That is, when the central Dirac delta function potential strength is $\Omega_{j}(N / 2+1) \pm 0.05 \Omega_{j}(N / 2+1)$. In Figure 6, we plot the electronic conductance spectrum for defected and defect free GTQW, both wires having the same strengths and the same number of Dirac delta function potentials,..., and scaling factor of three. As can noticed, there is a slight difference between the two conductance spectrum curves, which means we have a structure that can tolerate strength defect up to $5 \%$.

\subsection{Dislocation Defect}

In this subsection, we study dislocation defect effect on the position of the central element in the GTQW, and keeping the other elements and the spacing between the Dirac delta function potentials constant. First, we consider the position defect does not exceed $\pm 5 \%$ of the Dirac delta function potential spacing constant. That is, the central Dirac delta function potentials spacing is $d \pm 0.05 d$. In Figure 7, we plot the electronic conductance spectrum for both dislocations with $N_{j}$ is 17 and scaling factor of three. Compared to defect curves, as can noticed, there is a significant difference between the two curves. The conduction band starts lose its flatness and claims lower electric conductance values, but the forbidden band shape or width did not change appreciably for increased spacing between the central Dirac delta func- 


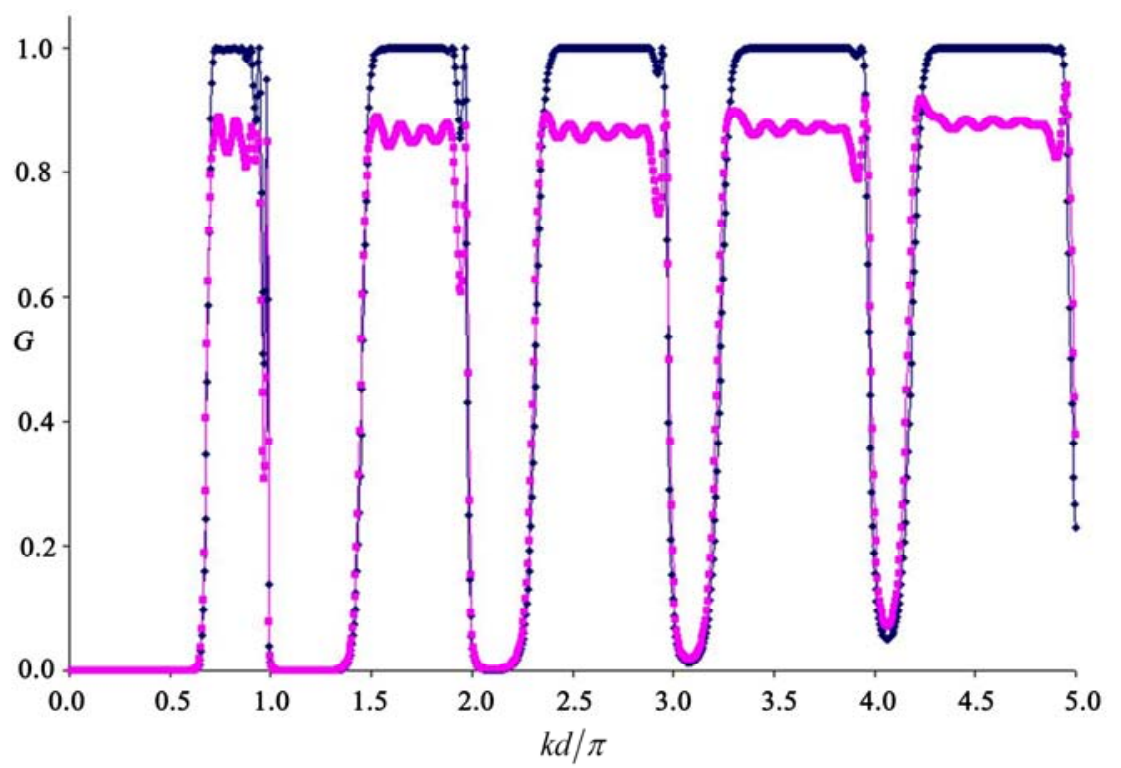

Figure 7. The electronic conductance, in the units of $2 e^{2} / h$ as a function of $k d / \pi$. In this case, the defect is only $\pm 5 \%$, in the position of the central Dirac delta function.

tion and the adjacent one.

\section{Conclusions}

On the Gaussian profile tailored quantum wire (GTQW), we have demonstrated numerically that these structures give rise to well-defined conduction and forbidden bands. The appearance of allowed conduction and forbidden bands is strongly dependant on the number of Dirac delta function potential in the distribution, and on its scaling factor. We found out that when the number of Dirac delta function increased in the Gaussian distribution, the conduction band gets wider, and the forbidden band gets sharper and narrower. When the strength of the Dirac delta function increased by a scaling factor in electronic conductance spectrum, it exhibited a well defined conduction band and forbidden band. We found that this novel structure has a good tolerance for strength reaches up to $\pm 5 \%$ and without losing the fascinating electronic transmission characteristics, but the structure is so sensitive to dislocation defect and its tolerance may not reach $\pm 5 \%$.

\section{References}

[1] D. Bolmatov and C. Y. Mou, "Tunneling Conductance of the Graphene SNS Junction with a Single Localized Defect," Journal of Experimental and Theoretical Physics, Vol. 110, No. 4, 2010, pp. 612-616. doi:10.1134/S1063776110040084

[2] M. Wimmer, M. Scheid and K. Richter, "Spin-Polarized Quantum Transport in Mesoscopic Conductors: Compu- tational Concepts and Physical Phenomena," arXiv:0803.3705v1, 2008.

[3] L. M. Jong, A. D. Greentree, V. I. Conrad, L. C. L. Hollenberg and D. N. Jamieson, "Coherent Tunneling Adiabatic Passage with the Alternating Coupling Scheme," Nanotechnology, Vol. 20, No. 40, 2009, p. 405402. doi:10.1088/0957-4484/20/40/405402

[4] G. Ariyawansa, S. G. Matsik, A. G. U. Perera and X. H. $\mathrm{Su}$ and P. Bhattacharya, "Tunneling Quantum Dot Sensors for Multi-Band Infrared and Terahertz Radiation Detection," Sensors, IEEE, 2007.

[5] Y. J. Doh, J. A. van Dam, A. L. Roest, P. A. M. Bakkers, L. P. Kouwenhoven and S. de Franceschi, "Tunable Supercurrent through Semiconductor Nanowires," Science, Vol. 309, No. 5732, 8 July 2005, pp. 272-275. doi: $10.1126 /$ science. 1113523

[6] N. P. Oxtoby, H. B. Sun and H. M. Wiseman, "Non-Ideal Monitoring of a Qubit State Using a Quantum Tunnelling Device," Journal of Physics Condensed Matter, Vol. 15, No. 46, November 2003, pp. 8055-8064. doi: $10.1088 / 0953-8984 / 15 / 46 / 020$

[7] H. Kajiura, H. Huang and A. Bezryadin, "Quasi-Ballistic Electron Transport in Double-Wall Carbon Nanotubes," Chemical Physics Letters, Vol. 398, No. 4-6, 11 November 2004, pp. 476-479.

[8] T. Nagahama, H. Saito and S. Yuasa, "Hot Electron Transport in Magnetic Tunnel Transistors with an Epitaxial Mgo Tunnel Barrier," Applied Physics Letters, Vol. 96, No. 11, 2010, p. 112509. doi:10.1063/1.3360222

[9] G. F. Ross and R. M. Mara, "Coherent Processing Tunnel Diode Ultra Wideband Receiver," United States Patent 5337054, 1992.

[10] D. W. L. Sprung and H. Wu, "Scattering by a Finite Periodic Potential," American Journal of Physics, Vol. 61, 
No. 12, December 1993, p. 1118. doi:10.1119/1.17306

[11] D. Kiang, "Multiple Scattering by a Dirac Comb," American Journal of Physics, Vol. 42, No. 9, 1974, pp. 785-787. doi:10.1119/1.1987841

[12] P. S. Deo and A. M. Jayannavar, "Quantum Waveguide Transport in Serial Stub and Loop Structures on," Physical Review B, Vol. 50, No. 16, 1994, pp. 11629-11639. doi:10.1103/PhysRevB.50.11629

[13] M. Sharma, S. X. Wang and J. H. Nickel, "Inversion of Spin Polarization and Tunneling Magnetoresistance in Spin-Dependent Tunneling Junctions," Physical Review Letters, Vol. 82, No. 3, 1999, pp. 616-619. doi:10.1103/PhysRevLett.82.616

[14] W. G. Wang, C. Ni, G. X. Miao, C. Weiland, L. R. Shah, X. Fan, P. Parson, J. Jordan-Sweet, X. M. Kou, Y. P. Zhang, R. Stearrett, E. R. Nowak, R. Opila, J. S. Moodera and J. Q. Xiao, "Understanding Tunneling Magnetoresistance during Thermal Annealing in Mgo-Based Junctions with CoFeB Electrodes," Physical Review B, Vol. 81, No. 14, 2010, pp. 144406. doi:10.1103/PhysRevB.81.144406

[15] H. S. Ashour, A. I. Ass'ad, M. M. Shabat and M. S. Hamada, "Electronic Conductance in Binomially Tailored Quantum Wire," Microelectronic Journal, Vol. 37, No. 1, 2006, pp. 79-83.doi:10.1016/j.mejo.2005.06.018

[16] H. Wu, D. W. L. Sprung, J .Martorell and S. Klarsfeld, "Quantum Wire with Periodic Serial Structure," Physical Review B, Vol. 44, No. 12, 1991, pp. 6351-6360. doi:10.1103/PhysRevB.44.6351

[17] W. D. Sheng and J. B. Xia, "A Transfer Matrix Approach to Conductance in Quantum Waveguides," Journal of Physics Condensed Matter, Vol. 8, No. 20, 1996, pp. 3635-3645. doi:10.1088/0953-8984/8/20/009

[18] T. Kostyrko, "Transfer-Matrix Approach for Modulated Structures with Defects," Physical Review B, Vol. 62, No. 44, 1999, pp. 2458-2465.
[19] E. Merzbacher, "Quantum Mechanics," Wiley, New York, 1970.

[20] H. Fayad and M. M. Shabat, "Electronic Transmission through Qunatum Wire Containing Hetero-Junction," Islamic Journal of Gaza (Natural Sciences Series), Vol. 13, No. 2, 2005, pp. 203-211.

[21] H. Fayad, M. M. Shabat, H. Khalil and D. Jäger, Proceeding of the IEEE Electron Devices Society, IEEE, Vol.11, 2001, p. 91.

[22] H. Fayad and M. M. Shabat, "Electronic Conductance through Some Quantum Wire Structure," Romanian Academy, Eds., Micro and Nano-Engineering Series, Nano science and Nano engineering, Dan Dascalu and Irina Kleps, Bucharest, 2002.

[23] D. W. L. Sprung, H. Wu and J. Martorell, "Periodic Quantum Wires and Their Quasi-One-Dimensional Nature," American Journal of Physics, Vol. 61, 1993, p. 1118. doi:10.1119/1.17306

[24] L. D. Landau and E. M. Lifshitz, "Quantum Mechanics," Pergamon Press, Oxford, 1976.

[25] G. Baym, "Lectures on Quantum Mechanics," W. A. Benjamin Incorporated, Massachusetts, 1973.

[26] Y. Takagaki and D. K. Ferry, "Electronic Conductance of a Two-Dimensional Electron Gas in the Presence of Periodic Potentials," Physical Review B, Vol. 45, No. 15, 1992, p. 8506-8515. doi:10.1103/PhysRevB.45.8506

[27] S. J. Blundell, "The Dirac Comb and the Kronig-Penney Model: Comment on 'Scattering from a Locally Periodic Potential,' by D. J. Griffiths and N. F. Taussig, American Journal of Physics, Vol. 60, 1992, pp. 883-888," American Journal of Physics, Vol. 61, No. 12, 1993, pp. 1147-1148. doi:10.1119/1.17312 